\title{
During Pandemic COVID-19, the High-Reliability Organization (HRO) Identifies Maladaptive Stress Behaviors: The Stress-Fear-Threat Cascade
}

Daved van Stralen, MD, FAAP, Thomas A. Mercer, RAdm, USN

\begin{abstract}
:
Stress has become an organizational characteristic. Organizations work toward stress reduction, seek stress management, increase cognitive capacity, attend to our mindfulness, and refer staff for anger management. The HRO acknowledges that stress, fear, and threat are natural elements of dangerous work. They are integral to and support actions when we do not know how events will resolve. Rather than compartmentalizing stress fear and threat, the HRO harnesses these driving forces to initiate engagement and support the resilience necessary to maintain enactment. For this to occur, we must identify unrecognized stress for its deleterious effects on performance. As we accomplish this, the organization can harness this energy to resolve the original stimuli while achieving an acceptable, if not desirable, end-state. The stress-fear-threat cascade enables us to engage uncertainty and threat, but without neuromodulation, the same cascade can cause more damage than the inciting event.
\end{abstract}

\section{"The stress-fear-threat cascade enables us to engage uncertainty and threat, but without neuromodulation, the same cascade can cause more damage than the inciting event."}

\section{Introduction:}

Stress can become one of an organization's characteristics, impeding reliability, creating unsafe conditions, and contributing to staff attrition. The "original" HROs operated where stress was endemic (van Stralen and Mercer 2020). The development of stress capacity in novices (Novaco et al. 1979) and staff (van Stralen and Mercer 2015) became integral to achieving reliability. On the other hand, maladaptive responses to stress, fear, and threat have a detrimental effect on safety and compromise reliability. Vigilance for these maladaptive stress responses is a form of preoccupation with failure.

The human stress system evolved to support an effective response against an acute physical life-threat, assisting immediate escape and survival (Hediger 1950, 19-20; Sapolsky 2004, 4-5; Lupien et al. 2007; Clinchy, Sheriff, and Zanette 2013). In modern times, acute stress can become sustained or chronic. Psychologi- cal stress "linked to mere thoughts" (Sapolsky 2004, 4-5) can sustain the release of stress mediators (Lupien et al. 2007; Clinchy, Sheriff, and Zanette 2013), causing structural changes in the brain (Lupien et al. 2007). We will discuss stress as an acute response because that describes the imminent danger for a person or the HRO. The chronic psychological stress reactions start with an acute reaction, becoming sustained when the individual cannot extinguish the acute phase of the stress reaction.
HROs accept the presence of stress and acknowledge the function of stress behaviors. The emphasis HROs place on stress capacity, psychological stability, and leadership (van Stralen, McKay, and Mercer 2020a, b) comes out of the purposes of the $\mathrm{HRO}$ characteristics and the fundamental reliance on the individual, a form of deference to expertise. This does not mean the $\mathrm{HRO}$ accepts or tolerates maladaptive stress or fear behaviors. It means that the HRO acknowledges that people (in the type of environments in which HROs operate) can become mentally or emotionally overtaken by abrupt, ambiguous events. The HRO, then, engages these behaviors as normal behaviors rather than weaknesses or moral shortcomings.

We have numerous first-hand or witnessed (DvS) accounts of widely respected physicians or surgeons treating a patient at the moment of abrupt deterioration. These doctors would freeze or rapidly and wordlessly begin acting. What these incidents have in common are barking, shouting, demanding orders given rapidly, without coordination or plan, often nonspecifically delivered to the general group. When one of the authors (DvS) presented such a case to a group of chief officers in the fire service, framed as a fire captain on the fireground, they unanimously said they would immediately remove the captain from duty with a referral for mental health assistance. When told that the scenario was a physician or surgeon, they reacted with astonishment.

NEONATOLOGY TODAY is interested in publishing manuscripts from Neonatologists, Fellows, NNPs and those involved in caring for neonates on case studies, research results, hospital news, meeting announcements, and other pertinent topics.

Please submit your manuscript to: LomaLindaPublishingCompany@gmail.com 
While the conserved stress system inhibits top-down cognitive control and enhances bottom-up reflexive actions, modulation by human executive processes can move mental processes toward effective cognitive flexibility. We describe the development of maladaptive stress behaviors in this paper.

'Sustained psychological stress' is a modern dysregulation of physiological and mental systems that had evolved for 'acute physical crises' (Sapolsky 2004, 4-5; Clinchy, Sheriff, and Zanette 2013). The constant work to maintain stability leads to chronic pathology. From a functional perspective, sustained stress feeds back to the individual's acute response, but in a negative way. Understanding acute stress functionality will refocus efforts away from preventing or mitigating all stress, limiting allostasis toward identifying dysfunctional stress, which contributes to allostatic load.

\section{"Functionally evolved for imminent physical danger, the stress-fear-threat cascade today reacts to thoughts and perceptions and can be modulated by thoughts and perceptions."}

Functionally evolved for imminent physical danger, the stressfear-threat cascade today reacts to thoughts and perceptions and can be modulated by thoughts and perceptions. A sense of controllability modulates stress responses. Fear, as a process of distance from threat, can drive adaptive actions or decompose to undirected offensive or defensive self-protection. Subcortical threat reflexes quickly initiate engagement or interfere with effective actions for self and others in a similar manner. This is not obvious; unrecognized stress, fear, and threat have become pervasive to the point of normalization in culture.

The authors' experiences in live-or-die situations illustrate that the stress-fear-threat cascade, when neuromodulated, is necessary for effective survival, safety, resilience, and reliability.

\section{From Perception to Stress}

\section{Perception to action}

Effective action responding to a changing environment integrates, from opposite ends of the brain, perception, hastily created plans, and motor activity. The dorsolateral prefrontal cortex (DPFC) and the posterior parietal cortex (PPC) functionally cooperate during time-based contingencies between continuous perception and emerging motor action (Quintana and Fuster 1999). Cognitive function for this to occur must include timing and coordination, the mode of information analysis of environmental events, and the temporal sequencing of the analytic processes as concurrent, reciprocal processing (Fuster 2000). The executive functions, acting hierarchically, coordinate temporary behavioral structures, and "integrate actions with perceptions in the presence of novelty and complexity" (Fuster 2000).

To maintain a working representation of circumstances, one must remember backward in time for several seconds to minutes and remember "forward" in time for "planned," prospective, near-future motor acts. This requires a timed element of acquiring, holding, and releasing information (Fuster 1999). Organizing behavior by time and timing, including the temporal closure of the perceptionaction cycle, sequences novel, and complex behavior (Fuster 1999). Working memory mediates perception and action in real- time (Fuster 1999).

A novel or complex environment contains distracting information and interference. The individual must protect goal-directed behavior from interference or impulsive or reflexive behavior (Fuster 2000) and inhibit emotional memories (LeDoux 2000; Joëls, Fernandez, and Roozendaal 2011), well-established habits, or more easily processed intuitions (Shtulman and Valcarcel 2012). The DPFC mediates internal and external stimuli to bring this inhibitory control (Fuster 2000).

\section{Executive Functions:}

The temporal organization of behavior, essential for sequencing novel and complex behavior, requires integration across time between prefrontal cortex representational neurons and posterior parietal cortex operant motor neurons (Quintiana and Fuster 1999; Fuster 1999, 2000). From lesion studies, brain cooling experiments, functional imaging, and electrophysiological studies, the authors identified specific roles for motor attention (impending motor action), working memory (sensory information for action), and inhibitory control (interference, impulsive and reflexive behavior). These three elements produce the operational control and temporal organization of behaviors that characterize the executive functions.

Complex cognition could control and organize behavior by any relatively independent processes or by a single process having multiple subfunctions. Akira Miyake et al. (2000) investigated the separability and contributions of the three generally proposed executive functions: mental set-shifting, information updating, and monitoring, and inhibition of prepotent responses. Using historical neuropsychological studies of patients with frontal lobe damage and current neuropsychological and cognitive studies, they characterized shifting mental sets, working memory, and inhibition as separable processes. They are not entirely independent, though, as they share related functions.

Using insights from developmental cognitive neuroscience, Adele Diamond (2013) showed a developmental progression in children. Further, the executive functions are effortful, though trainable, and can improve with practice.

This digression underscores the executive functions as separable, physiological processes with a developmental progression, rather than an esoteric academic construct. These functions, during the novel and complex circumstances, create logical reasoning and behaviors. In these circumstances, we use short-term memory of planned motor actions, memory to incorporate changing sensory information, adjustments to motor actions in progress, and inhibition of distracting information. We can easily lose the purpose of executive functions when we list them as three seemingly independent terms.

\section{Perception to stress}

Novelty, uncertainty, and uncontrollability, while in the domain of the executive function, can also cause stress (Mason 1968; Gagnon, and Wagner 2016). Novelty is processed in the right cerebral cortex, while the left cerebral cortex processes familiar perceptions. Uncertainty and ambiguity in decision-making occur in the ventromedial prefrontal cortex (vmPFC). Uncontrollability or unpredictability is the stimulus for the HPA axis.

The amygdala detects conflict from acute threats or stressors, receiving exteroceptive stimuli (the external environment) and interoceptive stimuli (the body's internal environment). The amygdala activates the sympathetic-adrenal-medullary (SAM) axis for the proverbial "flight-or-fight" response and the hypothalamic-pituitary-adrenal (HPA) axis for the release of peripheral adrenal 


\begin{tabular}{|l|l|l|l|}
\hline Authors & Fuster & Miyake et al. & Diamond \\
\hline Purpose & $\begin{array}{l}\text { Temporal, functional organization of } \\
\text { behavior }\end{array}$ & $\begin{array}{l}\text { Separable } \\
\text { Not completely independent }\end{array}$ & Developmental progression \\
Representative measures
\end{tabular}

hormones, including cortisol (Shields, Sazma, and Yonelinas 2016). The brain, reacting from bottom-up reflexive and priming processes, prepares the body for survival.

The rapid response of these directed, top-down responses gives the appearance of instinctive behaviors. Interpreting learned fixed action patterns, triggered by reflex, as instinct is a common misinterpretation. The presumption of instinctive behaviors dismisses salience, interpretation, and goal-directed behaviors, adding to the sense of uncontrollability.

\section{"The presumption of instinctive behaviors dismisses salience, interpretation, and goal-directed behaviors, adding to the sense of uncontrollability. "}

Uncontrollability alone causes minor stress, impairing the executive functions (Arnsten 2009). If unrestrained neurological stress responses develop, then almost pure bottom-up control and selfpreserving behaviors occur. Cortisol and the amygdala increasingly suppress the executive functions, and a defense cascade follows (Kozlowska 2017). Threats that are proximal (static distance) or approaching (changing distance) will mobilize one to move toward safety or, if escape is not possible, to fight in selfdefense.

Impaired executive functions, particularly working memory, are easily unidentified as we use our executive functions to evaluate our executive functions ("we use our judgment to judge our judgment"). Subordinates are then more likely to accept a leader's impairment as normative. Working memory manipulates the "chunks" of information that we can retain in short-term memory, limited to 4-7 chunks (Cowan 2001). The characteristics of these chunks contribute to the variance in number, whether due to temporal or spatial properties, the nature of the content, segregating into groups or integrating to a group, coherence of patterns, and capacity limits with information overload (Cowan 2001).

An unrecognized contribution to uncontrollability, given the facts above, is the presence in high-risk environments of compliance, rules, or linear protocol for engaging the embedded problem (van Stralen 2020). These become chunks of information readily reducing short-term memory by half and influencing, if not redirecting, working memory away from the problem at hand, moving toward an "accepted" response.

The author (DvS) sent a PICU transport team to a local medical center for an infant in cardiac arrest, over four hours intermittently responding but never stable. A second-year pediatric resident responded with the team, which successfully resuscitated and stabilized the infant twenty minutes after arrival. The resident sensed herself entering the attentive freeze response and, from the author's lectures, recognized it as neurochemical. She immediately examined the endotracheal tube, which brought immediate relief from her freeze sensation.

Stress responses also affect the salience of external and internal stimuli, influencing perception with changes in selective or focused attention, ultimately altering cognition. An immediate proximal but moderate threat can prime an array of reflexive, subcortical behaviors focused solely on one's survival. The acute effects of stress on perception can continue after the episode. Memory consolidation occurs after an event, making it amenable to cognitive edits from the individual or comments from bystanders or authority figures.

Stress is a measure and consequence of uncontrollability while at

\begin{tabular}{|c|c|c|c|}
\hline Cognitive-motor & $\begin{array}{l}\text { Motor attention } \\
\text { Representation of plan } \\
\text { Impending motor action } \\
\text { Motor memory for execu- } \\
\text { tion }\end{array}$ & $\begin{array}{l}\text { Shifting } \\
\text { Complex tasks } \\
\text { Mental set shifting between } \\
\text { multiple tasks } \\
\text { Disengage from an irrelevant } \\
\text { task set }\end{array}$ & $\begin{array}{l}\text { Cognitive flexibility } \\
\text { Exploit sudden, unexpected opportunities } \\
\text { Adjust to new demands, priorities } \\
\text { Change perspectives, approaches spatially, } \\
\text { interpersonally } \\
\text { Switch between tasks } \\
\text { Admit when wrong }\end{array}$ \\
\hline Information & $\begin{array}{l}\text { Working memory } \\
\text { Sensory information for } \\
\text { action }\end{array}$ & $\begin{array}{l}\text { Updating } \\
\text { Information updating } \\
\text { Manipulate relevant information } \\
\text { Monitor working memory }\end{array}$ & $\begin{array}{l}\text { Working memory } \\
\text { Mentally work with information: } \\
\text { - Held in mind } \\
\text { - No longer perceptually present }\end{array}$ \\
\hline Controllability & $\begin{array}{l}\text { Inhibitory control } \\
\text { Interfering information } \\
\text { Impulsive, reflexive behav- } \\
\text { ior }\end{array}$ & $\begin{array}{l}\text { Inhibition (deliberate) } \\
\text { Dominant, prepotent responses }\end{array}$ & $\begin{array}{l}\text { Inhibitory control } \\
\text { Attention, behavior, thoughts, emotions } \\
\text { Dominant, prepotent mental representations }\end{array}$ \\
\hline
\end{tabular}


the same time, stress promotes allostasis. After an abrupt change in circumstances, we must reset what we have learned and possibly change how we learn. Stress from a functional perspective tells us we need to improve performance and sustain our capacity if stress is sustained. Stress from an evolutionary perspective keeps the animal alive long enough to learn, then to be able to use that learning. Allostasis describes achieving stability through change. Continuous effort to achieve allostasis or allostatic load can create chronic pathophysiology and sustain psychological stress (McEwen and Wingfield 2003). Stress becomes a demand we have yet to meet.

"If we understand stress, fear, and threat as suites of behaviors, part of a person's response to the environment, we can understand the logic in their grouping and how they derive from, and form, a person's temperament. Temperament, as the affective, activational, and attentional core of a person, represents a person's reactivity (excitability, responsivity, and arousability) and self-regulation (Rothbart and Derryberry 1981; Rothbart and Bates 2007)."

Top-down modulation harnesses cognitive flexibility for rapid improvisation during abrupt, fluctuating change. Without recognizing how stress impairs perception, cognition, and behavior, we risk conserving maladaptive responses and not trusting our improvisations.

\section{Stress as Controllability:}

Acute stress functionally inhibits the executive functions through cortisol, the stress hormone in the hypothalamic-pituitary-adrenal (HPA) axis, and the amygdala in the sympathetic-adrenal-medullary (SAM) axis. Cortisol blocks memory retrieval in the prefrontal cortex and hippocampus (memory center), and the amygdala directly inhibits the prefrontal cortex.

Researchers remove or impair the volunteer's or animal subject's ability to control the experimental intervention to measure the effects of stress. The Yerkes-Dodson Curve (Yerkes and Dodson 1908) came about from studies increasing an electrical stimulus while measuring white-black discrimination in dancing mice. Their third and fourth conclusions were: 3 ) with easy discrimination, the dancers learned as the electrical stimulus increased, and 4) when "discrimination is extremely difficult," learning is rapid as the stimulus increases until a threshold is reached, then it decreased. The dancers were not the ones controlling the electrical stimulus.

Fire department Rescue Ambulance medics, working in a high crime area, would gain protection by asking the strongest appearing male to hold a roll of gauze for them. The individual gained a sense of control and participation. If violence threatened, he would intervene to protect "his" team, the medics. Similar control was observed in the PICU when anxious doctors adjust a knob on the ventilator, not realizing the knob simply changed the display.
They would studiously look at the child's chest, turn the knob, look at the display, look at the chest, and turn the knob again. This was similar to the "producer's knob" in a recording studio, not connected to anything, that would thwart "non-knowledgeable" participants from detrimental influence (Bates 2019). In experimental situations simulating the danger of combat, those recruits who remained at their post had focused on repairing a 'nonfunctional" radio needed for evacuation while those who self-evacuated had stopped working on the radio (Berkun et al. 1962). Through a sense of controllability, simple physical acts keep a person engaged and performing with the team.

Following the Yerkes-Dodson Curve, immediate life-threat would decrease a person's performance. That has not been our experience in live-or-die circumstances. Pragmatic HRO develops controllability in staff by strengthening their capabilities and stress capacities, thereby mitigating performance deficits due to stress. Authority can then migrate to those with expertise, allowing them to act. Deference to expertise, leading to action, promotes controllability to a greater degree than merely listening.

Impairment of the prefrontal cortex also impairs abstract thought. A sensitive indicator of a stress response, almost quantitatively, is the appearance of concrete thinking. "How much do the people on the scene think like a child. That tells you how serious the call is." This was one of the first ambulance lessons the author (DvS) received in 1972 . The more serious the call, the more concretely people think without conditioning and modeling by experienced service veterans (defined in the fire service by comparing "the person who does the same thing every year for 20 years versus the person who does something different every year for five years"). This situation is also true in critical and emergency care. Berkun et al. (1962) placed well-trained soldiers, most with combat experience, into the previously described study. These soldiers performed better than recruits, as expected, but differed from recruits in that the experienced soldiers performed better under the stress conditions than control.

Alfred North Whitehead (Whitehead, 1926/1967, 64) described "the Fallacy of Misplaced Concreteness" - the "error of mistaking the abstract for the concrete" - accepting abstractions "as the most concrete rendering of fact." Excluding the environment and context to make abstract concepts measurable supports the illusion of concepts as concrete reality and abstractions as concrete facts. We hear this when people rely on abstract concepts, metaphors, and clichés. Concepts are images of reality; we must not mistake concepts for reality.

Even though intuitions and scientific thoughts are abstractions, the loss of executive functions means that intuitive thought is not inhibited. Because intuitive responses are mentally faster than scientific responses, childlike misconceptions emerge, and the individual begins thinking in intuitions and superstitions. Inhibitory control is one of the executive functions. Without cognitive inhibition, intuitions predominate over scientific thought (Shtulman and Valcarcel 2012, 2016; Shtulman and Young 2019).

Stress is portable. Contrary to intuition, obtaining precise information and following rules and protocols is an unrecognized source of stress. Precise information becomes a goal, lacking feedback on its effectiveness. Accuracy requires feedback as we repeat goal-directed behavior to become more effective. Hence the artillery phrase, "ready, fire, aim!" (Dutch Army artilleryman, personal communication). Feedback augments, if not initiates, bottom-up influence. Managing feedback is critical for learning and reducing stress. Rules are, by their nature, discrete.

In the contingencies of the NICU, rules may overlap, compete, or conflict with the query of who chooses the rule. Protocols are de- 
terministic and linear, while, as noted above, our executive functions operate for the novel and routine situations of complexity where information changes and the effectiveness of actions are not known until the act. Even then, we cannot identify effectiveness versus mistake [or error] while the situation unfolds (Paget $1988,45)$. The pressure to predict drives the passive search for predictive information rather than active engagement, which will generate Shannon Information by converting uncertainty to certainty through action. This type of engagement, to generate information, creates order, structure, and predictability.

While the demands on leaders are greater than their subordinates, their sense of control from leadership can buffer against stress (Sherman et al. 2012). Unfortunately, this can be a draw for some leaders to increasingly retain control during a crisis, reducing their stress load at the expense of subordinates.

ilnhibition allows one to inhibit thoughts or prepotent responses, allowing selective attention to task-relevant information and engagement of goal-directed actions. Working memory refers to keeping information in mind and updating/integrating current content with new information. Cognitive flexibility refers to the ability to shift between cognitive rules or modes of thought (Lupien and Lepage 2001).

\section{Fear As Process:}

The pragmatic stance integrates the mental and behavioral effects of fear within the operations of HRO. We cannot predict anything about fear other than it will affect someone. More damaging to an HRO than the effects of fear are the unrecognized effects of fear on interpersonal interactions, behaviors, cognition, emotion, and attrition.

When a predator approaches, an animal responds to avoid the enemy with what Heini Hediger (1950, 19-20) called an "escape reaction." The animal does not run away but has a suite of behaviors to avoid the enemy (not all threats come from predators). The suite is characteristic for the animal, the species, the enemy, and the environment. If the enemy continues to approach and reaches a specific distance, the "flight distance," the animal will flee, trying as quickly as possible to regain the flight distance. During flight, the animal will not attack. If the enemy enters the "defense distance," the animal will attack but always with the character of emergency self-defense. When the enemy enters the "critical distance," the animal will attack with emergency characteristics, going beyond self-defense. This attack is associated with territoriality.

This departure into animal behavior illustrates fear as a threat to the significant characteristics of:

- Distance

- Bottom-up subcortical reactions

- Top-down cortical modulation

- Rapid behavioral shifts

Because fear reactions integrate stress and threat, we will discuss in this section fear as a reaction to a threat coming closer in proximity.

\section{Threats:}

The subjective nature of threat prevents us from predicting what a person will perceive as a threat or how they will respond to a threat. In this sense, threat in an HRO becomes a shared experience in that colleagues, particularly leaders, monitor each other and subordinates for early signs of stress or fear. For example, the nature of a person's humor reveals evidence of fear - humor as mastery, tension relief, or to redirect aggression - gives clues to the leader about a person's state (van Stralen, Byrum, and Inozu 2017, 291-3).
The subjective representation of threat, and the degree to which it is felt, is processed in the midbrain periaqueductal gray (PAG) nucleus. The PAG coordinates behaviors essential to survival, including threat reflexes, rapid changes to subcortical behaviors, and startle/posture corrections. The PAG also processes the proximity of threats (Mobbs et al. 2007).

Fear motivates a person to act in order to reduce the potential danger from a threat. While it is common to speak of predatorprey interactions, it is more useful to view fear as a motivating drive to protect one's physical, mental, and emotional body from attack or collision (Graziano and Cooke 2006). How we protect ourselves every day is how we protect ourselves in an extreme crisis or life-threat (Jim Denney, Vietnam Veteran, and Captain, Los Angeles Fire Department).

An unrecognized or misinterpreted threat may come from ventilator management. The intensive care unit properly focuses on blood gas derangements, treated with technical methods for oxygenation and ventilation. Overlooked is the effect of sensation. In a study to evaluate curare's central nervous system effects, a volunteer received curare stepwise until achieving full paralysis (Smith et al. 1947). "Shortness of breath" occurred "in the presence of adequate pulmonary ventilation and oxygenation" but resolved with "smooth rhythmical pressure... alternating with slow even release" at a slightly higher than normal rate. Curare had been administered to infants and children without additional medication (Smith et al. 1947), and a belief persisted that curare had some central depressant action and could enhance analgesic or anesthetic action (Kellgren, McGowan, and Wood 1946). The study also disconfirmed the motor theory of voluntary thinking, that muscle activity was necessary for perception and thinking (Cohen 1986).]

Subcortical fear responses or threat reflexes can occur from the sense of suffocation in the presence of adequate oxygenation and ventilation. Any form of chest restriction, for example, COVID-19, acute asthma, or mechanical restriction, can induce physiological and psychological fear reactions despite sufficient ventilatory efforts for gas exchange and speech. With central neurological injury, a neonate in the NICU may, instead, show symptoms of hypoactive delirium (Groves, Traube, and Silver 2016). In a study of PICU patients, $17 \%$ had delirium with $46 \%$ hypoactive delirium (Traube et al. 2017). In two pediatric subacute facilities, pediatric patients with hypoactive or hyperactive delirium immediately calmed by hand ventilation alone, changing their diagnosis from persistent vegetative state to profound intellectual disability. Many began smiling and attending school (author's experience, DvS).

\section{Distance: Physical, Emotional, Mental, or Temporal:}

We maintain distance for safety. The distant threat, yet within the "flight distance" for physical, emotional, mental, or temporal threat, increases activity in the ventromedial prefrontal cortex (vmPFC), which connects to the amygdala for the determination of the motivational importance of or degree of, the threat. The vmPFC also incorporates contextual factors into decision-making, whether intrinsic or extrinsic to the organism or environmental factors (Fellows and Farah 2007). The amygdala connects onward to the bed nucleus of the stria terminalis (BNST) to control a repertoire of behavioral defensive states (Mobbs et al. 2007).

The additional proximal threat will switch activity from the vmPFC to the phylogenetically older midbrain, increasing PAG activity. The PAG controls fast reflexive behaviors (e.g., fight, flight, or freeze) and fear-induced analgesia (Graziano and Cooke 2006; Mobbs et al. 2007). The release of endogenous opioids in the PAG inhibits the effect of expected pain on decision-making. (The vmPFC is important for decision-making in uncertain, risky, ambiguous, or context-dependent conditions, Fellows and Farah 2007.) 
The PAG also identifies an approaching or receding threat (Adolphs 2013), specific to one of the greatest fears, an approaching predator. Detection of changes in distance from threat functionally switches the repertoire of behaviors the animal uses (Adolphs 2013). Berkun et al. (1962) found this from the descriptions of army recruits in dangerous situations. Distance as perceived physical proximity or time dominated the thinking of "evacuators," becoming the determinant for running away.

This movement from contextual decision-making under uncertainty in the vmPFC to reflexive decision-making from the PAG makes the fight or flight of the fear reactions appears to be the same as the fight or flight from threat reflexes. What it describes, though, is the functional flow of response to a developing danger as apprehension leads to avoidance (flight), then becomes engagement (self-defensive fight). As a functional approach, the fear reactions (PAG) develop from distance-based assessments, while threat reflexes (amygdala) come from active danger.

The PAG has different functions in its several dorsoventral and rostrocaudal divisions. Dorsal stimulation promotes passive freezing, while ventral stimulation promotes escape and other active coping behaviors (Mobbs et al. 2007). From nose to tail, active coping strategies shift from moderate threat display to active defense, then aggressive defense; then strong threat display and non-opioid-mediated analgesia; followed by vigorous escape when the enemy is near. When escape from an enemy is impossible, passive coping strategies disengage from the environment and behaviors shift to freezing, and then with increasing proximity, moderate to strong immobility. Lastly, strong freezing with opioidmediated analgesia occurs (Koutsikou, Apps, and Lumb 2017; Watson et al. 2013).

Social distance acts as either a threat or as support. The close physical proximity of a threatening person elicits the same reactions as any threat. Fear responses are also transmitted through social interactions. On the other hand, as social support, people create a protective factor against stress, reducing the hypothalamus-pituitary-adrenal axis responsiveness to social stress (BrillMaoz and Maroun 2016).

As favorable or unfavorable, social distance is subjective, but the peripersonal (i.e., near body) space is not. This is the space where intrusion by others elicits discomfort. This space is measurable in the encoding of the visual receptive fields involving the ventral intraparietal area (VIP) and a polysensory zone in the precentral gyrus (Lloyd 2009). Responses are sensitive to nearby or approaching objects (Graziano and Cooke 2006). The VIP connects to the amygdala, then to the PAG for defensive and aggressive behaviors (Lloyd 2009). The neuropeptide oxytocin partly mediates social interaction and may also regulate fear in these situations (Brill-Maoz and Maroun 2016).

\section{Flexibility:}

The continuous switching within the PAG is a blend of the bottomup responses to threats before they come to our awareness and top-down cortical neuromodulation from the vmPFC and the anterior cingulate cortex. This switching supports rapid behavioral shifts from the threat reflexes.

Fear reactions are conscious sensations experienced when exposed to an imminent threat (Panksepp, Thomas, and Paolo 2011; Ledoux and Pine 2016). The amygdala sends signals to the unconscious (subcortical) and conscious (prefrontal cortex) regions of the brain, accounting for the uncontrolled fear responses and the feeling of fear. The emotional response of fear, preceded by a threat to self-preservation, diminishes danger (Oatley \& JohnsonLaird 2014). This creates the drive to avoid or escape, generally focusing on self-interest, self-protection, or the protection of oth- ers. We can regulate the feelings of fear by reappraising the situation or suppressing the behaviors (Ochsner and Gross 2008; Heilman et al. 2010; Cutuli 2014; Gross 2014, personal experience of the authors).

Actions for offensive protection, often developed from a developed plan, take the individual into a prompt attack to stop the problem's spread. The aggressive projection of force secures the initiative but becomes pathological when directed at people. The person will use surprise, concentrated actions, fast tempo, and audacity. They will use blame, accusation, and personal attacks.

Actions for defensive protection focus on the individual's safety, often with the person moving to a place of psychological or physical safety (Oatley \& Johnson-Laird 2014). Any ad hoc emergency plan is singularly focused on personal survival or a sense of safety. The person enters this defensive mode when demands clearly exceed capabilities, and risks become too great for them to feel they can continue or survive. The person will not go near the threat source, which could be the leader, an administrator, or a colleague. Rationalizations and abstractions (for example, clichés and metaphors) support actions since the individual has not approached sufficiently close to the situation to identify correlations or causations. This individual is less useful to protect others because the focus is primarily to reduce risk to themselves. The person will deflect, excuse, justify, or use prophylactic self-blame.

\section{Threat as Reflex:}

Fight, flight, and freeze are commonly referred to as the "fear responses." For the functional reason previously mentioned, we distinguish between the cortical "fear responses" due to distance from a threat and the subcortical "threat reflexes" due to attack, that is, the imminence of a threat. We cannot control the threat reflexes as a reaction, but we can inhibit sustained threat reflexes and control consequent behaviors. The term "instinct" is commonly used to describe the reflexive response and associated pattern of action. This disregards the associated learned behaviors, called "fixed action patterns," that form mammalian "instinctive behavior."

The wide repertoire of behaviors observed and experienced derives from a few mammalian defensive behaviors. Conscious, top-down modulation of the nonconscious, reflexive motor and emotional elements generate this complexity. Therefore, the behavioral or emotional component of the reflex may not occur. For example, the fight response without the behavioral component appears as anger. The threat responses, with or without both components, will continue to impair cognition. A sense of uncontrollability enhances the appearance of maladaptive consequences. Uncontrolled defensive behaviors are brittle and imperil survival. Even the perception of control is sufficient to diminish these reflexive behaviors.

Threat reflexes initiate behaviors for survival and adaptation to adverse or hostile environments. Perceptions of threat trigger reflexes that operate below the level of consciousness (LeDoux 2014).

- Fight engages in overcoming the threat.

- Flight rapidly increases separation to the flight distance previously described.

- Freeze, as attentive awareness with the cessation of movement, has two components: 1) focused collection of the necessary information and 2) posture poised for immediate, effective action.

- Tonic immobility, the intense awareness with the inability to move, is accompanied by mild-to-severe nausea and possibly evacuation of body contents. 
Three other threat responses bear discussion: startle reflexes, dissociation, and emotional memory.

- Startle reflex for defensive posture, gait and postural responses, and voluntary escape movements.

- Dissociation occurs when abrupt, unexpected, overwhelming events depersonalize an individual and fragment memory consolidation.

- Emotional memory develops from a single, emotionally charged incident, preparing the individual for a similar circumstance.

How they present:

- Fight is manifested by anger and frustration

- Flight takes the form of avoidance and distraction.

- Freeze as a physical freeze is an immobility with intense attention, while a mental freeze is the inability to recall knowledge or use working memory.

- Tonic immobility, despite awareness of surroundings, prevents physical movement, but milder presentations are intense aversion, gastric upset, or nausea.

- Startle reflex scream, an involuntary jerk, or "start."

- Dissociation is depersonalized, emotional numbing.

- Emotional memory presents as a severe response independent of and disproportional to the event.

Fight. As described by Hediger (1950, 20), an animal will attack with emergency characteristics, going beyond self-defense, when an enemy enters the critical flight distance. The separation of the motor and emotion components leads to responding with anger (emotion component) without physical contact (motor component).

The prevalence and pervasiveness of relaxed fight responses give the impression that anger is normal, if not necessary, behavior in an urgent or emergency environment. The immediate reactions observed by the use of the fear responses of anger and force, for example, reinforce the belief in their effectiveness. However, the observed effectiveness is an immediate change toward homeostasis at best, while impairing allostatic strengthening.

Flight. As described in "The Process of Fear" above, the animal flees due to an enemy's proximity. Fight during the fear process enables escape (Hediger 1950, 20).

In human terms, the person will "flee" due to the motor component to physically leave or display the emotional component and avoid, ignore, or distract, perhaps by asking for more information (McConnell and van Stralen 1995). Verbal maneuvers include denial, dismissiveness, or depreciation of disconfirming information.

Attentive freeze. The body is tense and poised to act; the mind is watchful, collecting information. In prey species, it prevents motion detectable by predators. Freeze is the brake on fight-or-flight reactions to learn more, avoid a fight, or prevent futile flight to failure. Freezing is also associated with faster subsequent cuesignaled responses (Roelofs 2017)

Information can have more than one meaning, and actions can have more than one effect, contributing to the hypervigilant freeze. This pause can be misinterpreted as denial, indecision, confusion, or waiting for leadership.

Tonic immobility. The person is "frozen" and, despite muscle tone, cannot move (differing from attentive freeze), emotionally aroused, full of fear, unable to call out, or respond to pain. Yet the person maintains full awareness and consciousness (Abrams et al. 2009; Kozlowska et al. 2015). The vagus nerve mediates many of the tonic immobility features: bradycardia (slow heart rate), lifethreatening arrhythmias, decrease in respiration, nausea and vomiting, urination, and defecation.

Without the behavioral component, tonic immobility appears as the feeling of nausea when faced with a difficult decision, the "pit of my stomach" feeling. For novices, nausea accompanies their first independent decision and, if not resolved, will inhibit future decision-making. The individual does not necessarily become trapped in tonic immobility. Kozlowska et al. (2015) described actions a Second World War Flying Officer would take when training pilots: he used a "firm voice devoid of fear to issue simple orders that the men had already learned and that was automatic: 'flaps,' 'raise the stick,' 'rudder."'

Startle reflex. A stumble, a sudden, loud sound, or a quick movement noticed from the corner of your eye, requires reflexive protective action. With rapid body movements, one regains balance, reflexively postures to protect vital organs, and becomes poised for action. Mentally, one assesses information for salience, meaning, and relevance (Valls-Solé et al. 1999; Nonnekes et al. 2015; Shemmell 2015). Through convergent evolution, startle became a repertoire of protective behaviors reflexively, bringing protection from disconnected threats represented by sound, sight, and imbalance. More commonly, they present as a single scream (for example, in a scary movie), flexing into the fetal position for protection during a fall (Bakker et al. 2006) or suddenly attending to a "distraction."

Vocalizations in the startle response may be misinterpreted as "screaming in panic" when they are actually an involuntary reflexive response to regain posture, orient toward a threat, and prepare for voluntary movement.

Dissociation. Fragmented awareness and derealization, producing an oddly calm but flaccid, vague stare, an emotionally numb individual, can appear during objectively innocuous situations. Though this dissociative response is more likely when circumstances overwhelm, realities suddenly become intolerable, or intense emotions are experienced (Frewen and Lanius 2006; Lanius, Paulsen, and Corrigan 2014). You may have observed this flaccid look of absence at the moment circumstances disrupt and fragment the individual's worldview, even when the individual has experience and authority.

Though it appears the person does not care, it is better explained as dissociation. Dissociation can occur as detachment, described above, or by compartmentalization, when controllable processes or actions become separated, continuing to operate without conscious control. The person functions inappropriately for the circumstances (Holmes et al. 2005). Dissociation may be more common than we realize, such as the individual receiving overwhelming information or the leader receiving information that disconfirms strongly held beliefs.

Emotional memory. Novices entering a dangerous field have all felt the sudden sting of the "old timer's" wrath, seemingly out of proportion to the minor act. Those in the work environment, immersed in events, have experienced the abrupt, unexpected, abject failure without recovery. Both authors have heard and lived with the understated phrase, "Well, we don't want to do that again." "That" likely being injury or death during operational activity. While the memory of events may fade, similar indices will, through the amygdala, trigger a life-preserving response. With meaning-giving and insight, this becomes adaptive. With isolation or blame, this becomes pure destruction. The HRO gives meaning and shares insight. No one is allowed to be isolated. No one is to blame. 
Strong emotional experiences, particularly those associated with threat, encode into a type of memory that is readily triggered by closely similar circumstances and that does not extinguish with time (LeDoux 2000; Joëls, Fernandez, and Roozendaal 2011). Rarely does memory recall come in the form of the person reexperiencing the event, known as a 'flashback.' More likely, the current circumstance elicits sensations associated with the originating experience. The emotional memory directly and unintentionally takes over redirection of attention. Neonatology, as a field of practice, is quite associated with emotional memory.

\section{Relations Between Fear-Stress-Anxiety-Threat:}

Behaviors and feelings emerge from nonlinear, complex neurological interactions between several orthogonal systems. Novel and familiar perceptions enter the right or left cortex, respectively. Some people are comfortable with novelty; some are not. Novelty can trigger the HPA system to release cortisol. Decision-making under uncertainty occurs in the vmPFC. Uncertainty can trigger the HPA system to release cortisol. Events in flux can be uncontrollable, another releaser of cortisol from the HPA system. As an enemy comes closer, spatially or temporally, the origin of behaviors moves from the VmPFC to the PAG, where self-defensive behaviors begin to predominate. Cortisol, released due to novelty, uncertainty, or uncontrollability, begins to inhibit memory systems, drawing focus on learned behaviors rather than cognition. At some point, the threat becomes imperiling, and threat reflexes predominate. Cortisol release interferes with cognitive neuromodulating influence. Isolating the brain laterality, the HPA axis, and the SAM axis makes sense for research and developing models but misleads when attempting to understand and observe behaviors during an incident. Confounding factors include perceptions, experience, and social support, whether convergent, local, or from leaders.

\section{Comparison of fight and flight between fear and threat}

Fight or flight, as offensive and defensive fear responses or as threat reflexes, have different timelines, stimuli, and purposes. "Fear flight" begins more slowly, mediated by the PAG, while the enemy is at a distance from the individual, initiating the movement to regain the "flight distance." Threat flight occurs immediately when a sudden immediate threat stimulates the amygdala and SAM's survival behavior. Physically, the person in "fear flight" has impaired cognition (cortisol) and escapes directly toward a safe place. In "threat flight," the individual retains cognition, begins evasive actions, and maneuvers away from the threat.

Flight due to fear. As a fire paramedic, the author (DvS) responded to a shooting in a city park during a friendly neighborhood touch football game. Arriving fairly shortly after the event, people were uncharacteristically distant from the victim. At close range, a shotgun round removed a section of the victim's rib, exposing his lung. The victim was deceased. A few bystanders reported an adolescent, a second victim, had run home. Reaching this victim in his house, he frantically repeated that he tried to be good, go to school, do his homework, stay away from gangs. With shotgun pellets in his body, he had run directly to a safe place and sat his home. Flight as an unengaged fear response, without neuromodulation, drives the individual to escape to a safe zone rapidly.

Flight due to threat. As a US Navy Aviator flying over North Vietnam early in the war, the author (TAM) routinely encountered gunfire and surface-to-air missiles. Unless you were in the target area, you may not know or be concerned about its source and direction. You countered with normal jinks (random turns to counter the firing solutions) and continued to the target in a random direction. You could often visually spot a SAM missile lifting off, a new method of attack that even the Korean War veterans $(\mathrm{CO} /$ $\mathrm{XO}$ ) in the squadrons had not experienced. Your primary counter- measure was mild maneuvering to determine whether the missile was tracking toward your aircraft. If it was, you maneuvered to keep it in sight and continued jinking until it was close enough that the missile was very close, then you turned hard into it. With command detonation from the ground, you could outmaneuver the big/fast missile, confounding efforts to detonate it. But with hard maneuvering, you lost altitude and airspeed, and the energy necessary to evade a second missile or remain above the intense gunfire at low altitude. Flight-as engaged threat reflex could dominate the action during the bombing mission, but damage could result without neuromodulation.

Fear fight, for self-defense, starts within the defensive distance, to help the individual escape. Threat fight, like threat flight, also occurs immediately in response to life-threat. George Williams, police tactics trainer, found that law enforcement officers experienced with street fights had no experience with THE FIGHT. That is the self-defense fight and is the more common way to escape. In THE FIGHT, the assailant wants to hurt the officer (van Stralen Byrum, and Inozu 2017, 257-9). Without the motor component, we can distinguish a self-defense argument, to escape with pride and ego intact, from the attack argument, the "assailant" wants to (emotionally or professionally) hurt the healthcare professional. Mismatched responses risk acceleration and loss of control. We use a different analysis level for the different systems (fear, threat), not different responses.

\section{Comparison of tonic immobility with vasovagal syncope}

The "common faint" occurs in an emotional context when the vagus nerve rapidly decreases blood pressure (Blanc et al. 2015). Complete loss of consciousness distinguishes vasovagal syncope from tonic immobility, where the person remains fully aware. Vasovagal syncope could, like tonic immobility, be an adaptation to mimic death (Bracha et al. 2005) or as a response to the sight of blood, injury, or injection. Syncope from seeing one's own blood may have adaptive value to reduce blood loss by rapidly decreasing blood pressure through vagus nerve activation (Diehl 2005).

\section{Comparison of attentive freeze with tonic immobility}

Freeze and tonic immobility responses appear similar to an observer but have distinct survival purposes. A person in the "freeze state" maintains attentiveness while remaining motionless, poised for action whether to initiate fight or flight. A person in tonic immobility maintains awareness and will create memories during this phase but cannot respond to stimulation.

\section{Comparison of dissociation and emotional memory}

During the experience of overwhelming, threatening circumstances, the individual may retain vivid memories (Kozlowska et al. 2015) or experience memory retrieval deficits (Schauer and Elbert 2010). In the hypervigilant state, a narrow range of stimuli may be sharply encoded (Allen, Console, and Lewis 1999). Emotional memory formation is closely linked to the amygdala and hippocampus (Murty et al. 2010), appearing to need timing with norepinephrine and cortisol release (Joëls, Fernandez, and Roozendaal 2011). On the other hand, during dissociation, the loss of context fragments the memory and impairs encoding the ongoing experience into memory. The dissociation of context and disrupted cortical integration prevent memory encoding (Allen, Console, and Lewis 1999).

\section{Unrecognized Stress, Fear, Threat:}

We tend to think of a fear response as something physical, such as the fight-or-flight response. Most people do not recognize anger or avoidance as fear responses. We also tend to see confusion and fear as weakness or failure. These are all fear responses 
mediated by neurochemicals. They can come on with incredible speed and, when accepted as simple neurochemical effects, can be interrupted almost as quickly.

The unrecognized fight responses include anger and frustration. One author (DvS) routinely queried staff, "What would make an attending angry with you?" Answers focused on errors or poor performance. After learning about stress, fear, and threat, the answers changed - "The attending is in a fear response or threat reflex." The significance lies in the subordinate's response; becoming more careful or working harder does not decrease fear. Asking "How can I help?" moves cognition from the amygdala to the prefrontal cortex.

The unrecognized flight is manifested by aversion, plausible avoidance, distraction, delay, and denial behaviors. Unrecognized attentive freeze responses include mental freezing and confusion while maintaining awareness. Tonic immobility, as a sensation, gives an unsettled feeling in the stomach and commonly nausea. One author (DvS) found that nausea is commonly associated with the first independent decision a novice makes.

The behaviors we identify with unrecognized fear have become incorporated into today's culture as acceptable behaviors, if not norms, to be emulated. They arise from the well-known fear responses of fight, flight, and freeze but are redirected to more modulated behaviors than the "wild type" we would expect outside the workplace. Unrecognized fear behaviors include anger (including instrumental anger), avoidance, mistaken deference to authority, over-scrutiny of disconfirming information, and cognitive dissonance. We label them "unrecognized" because people all too commonly consider them accepted, or even normal, responses.

Failure develops either because of unrecognized fear responses or the instrumental use of fear or threat by someone. This failure may also be a failure to respond to signs or signals undetectable for some reason.

\section{Conclusion:}

Because the human brain evolved for the novel and complex, we see in the stress fear threat cascade the recruitment of specific aspects for our protection, for example, constrained memory, focused perception, or quick motor action. For survival on the savannah, the enemy must be far enough for security, a relatively objective distance. Flight distance and imminent harm in modern life, however, is subjective and contextual. Constant exposure to the enemy and conflict, with the continuous release of mediators, corrupts neuromodulation by the PFC and executive functions. The threat does not resolve, we cannot resolve the last decision, and the temporal closure of the perception-action cycle (Fuster 1999) does not happen. We do not receive our reward from the nucleus accumbens. We do not feel the internal signal that the enemy is gone, that we now have security. Organizational stress as a culture of stress generates a continuous release of mediators, priming perceptions to make the next threat more daunting. The threat, during an already aroused state, signals a dangerous environment. This is not HRO.

The hallmark of acute stress is cortisol release with inhibition of memory. Chronic stress also releases cortisol with a subtle, nuanced effect on the executive functions of inhibition, working memory, and cognitive flexibility. To say "we feared for our lives" or "is there liability" is to normalize these suites of behavior and normalize not neuromodulating stress responses. For an organization operating in chronic stress, a culture of stress, performance deficits are no longer noticed, if not becoming normative, and performance decrements are part of operations. This normalizes suites of behaviors derived from stress, fear, and threat. Learned ensembles of stress behaviors come into routine use. Burn out, the loss of meaning in work or value of our efforts, and staff attrition, a form of fear flight, become expected.

The drive to survive generates emotional energy that, somewhat like thermodynamics, can transform, but it must dissipate. This survival energy supplants the rewards of the nucleus accumbens or, through aggression, transforms stress into reward. The shortterm reward comes from changing the environment; even change that hurts or damages is good because it gives a sense of control. Unfortunately, this energy may be released as aggression, which, through immediate feedback, becomes reinforced. Even if the individual suffers or creates a challenging living environment, the sense of control over other people immediately reduces stress. Rationalizations and justifications alleviate stress between acute stress episodes.

Emotional survival energy also transforms into tissue damage, producing the long-term effects first identified by Selye. Freud described behaviors through evolution and the principles of thermodynamics when applied to procreative energy. We describe behaviors through survival and the principles of thermodynamics applied to stress behaviors.

The control of any form of energy contributes to productivity and

\begin{tabular}{|c|c|c|c|c|c|}
\hline Condition & Cause & Characteristics & Location & Effects & Resolution \\
\hline Stress & $\begin{array}{l}\text { Novelty } \\
\text { Uncertainty } \\
\text { Uncontrollability }\end{array}$ & $\begin{array}{l}\text { Objective } \\
\text { Neurochemical re- } \\
\text { lease }\end{array}$ & $\begin{array}{l}\text { SAM } \\
\text { HPA Axis, corti- } \\
\text { sol }\end{array}$ & $\begin{array}{l}\text { Impaired declarative, } \\
\text { working memory } \\
\text { Impaired cognition }\end{array}$ & Perception of control \\
\hline Fear & Proximity & $\begin{array}{l}\text { Subjective } \\
\text { Feeling }\end{array}$ & $\begin{array}{l}\text { Ventromedial } \\
\text { Prefrontal cortex } \\
\text { Periaqueductal } \\
\text { Gray }\end{array}$ & Maintain distance & Reframe \\
\hline Threat & Existential harm & $\begin{array}{l}\text { Objective } \\
\text { Behaviors }\end{array}$ & $\begin{array}{l}\text { Amygdala } \\
\text { Prefrontal cortex }\end{array}$ & $\begin{array}{l}\text { Fight, anger } \\
\text { Flight, avoid } \\
\text { Freeze, vigilance } \\
\text { Tonic immobility, nausea } \\
\text { Dissociation }\end{array}$ & Conditioning \\
\hline
\end{tabular}


safety, the principle behind HRO. When uncontrolled, accidents can become normal. This is the same regarding neuromodulation of the stress-fear-threat cascade. When neuromodulated, we can effectively and safely engage uncertainty or threat. Without neuromodulation, the same cascade can cause more damage than the inciting event.

\section{References:}

Abrams, Murray P., R. Nicholas Carleton, Steven Taylor, and Gordon JG Asmundson. 2009. "Human tonic immobility: Measurement and correlates." Depression and anxiety 26(6): 550-6.

Adolphs, Ralph. 2013. "The biology of fear." Current Biology 23(2): R79-R93.

Allen, Jon G., David A. Console, and Lisa Lewis. 1999. "Dissociative detachment and memory impairment: Reversible amnesia or encoding failure?" Comprehensive Psychiatry 40(2): 160-71.

Arnsten, Amy F. 2009. "Stress Signaling Pathways That Impair Prefrontal Cortex Structure and Function." Nature Reviews Neuroscience 10 (6): 410-22.

rBakker, Mirte J., J. Gert Van Dijk, Arn MJM van den Maagdenberg, and Marina AJ Tijssen. 2006. "Startle syndromes." The Lancet Neurology 5(6): 513-24.

Bates, Eliot. 2019. "Technological Encounters in the Interculturality of Istanbul's Recording Studios." El Oído Pensante, 7 (1): 145-71.

Berkun, Mitchell M., Hilton M. Bialek, Richard P. Kern, and Kan Yagi. 1962. "Experimental studies of psychological stress in man." Psychological Monographs: General and Applied 76(15): 1-39.

Blanc, Jean-Jacques, Paolo Alboni, and David G. Benditt. 2015. "Vasovagal syncope in humans and protective reactions in animals." Europace 17(3): 345-9.

Bracha, H. Stefan, Adam S. Bracha, Andrew E. Williams, Tyler C. Ralston, and Jennifer M. Matsukawa. 2005. "The human fear-circuitry and fear-induced fainting in healthy individuals." Clinical Autonomic Research 15(3): 238-41.

Brill-Maoz, Naama, and Mouna Maroun. 2016. "Extinction of fear is facilitated by social presence: Synergism with prefrontal oxytocin." Psychoneuroendocrinology 66: 75-81.

Clinchy, Michael, Michael J. Sheriff, and Liana Y. Zanette. 2013. "Predator-induced stress and the ecology of fear." Functional Ecology 27(1): 56-65.

Cohen, Barry H. 1986. "The motor theory of voluntary thinking." In Consciousness and self-regulation: advances in research and theory, edited by Richard J. Davidson, Gary E. Schwartz, and David Shapiro 19-54. New York, NY: Plenum Press.

Cowan, Nelson. 2000. "The magical number 4 in short-term memory: A reconsideration of mental storage capacity." Behavioral and brain sciences 24(1): 87-114.

Cutuli, Debora. 2014. "Cognitive reappraisal and expressive suppression strategies role in the emotion regulation: an overview on their modulatory effects and neural correlates." Frontiers in systems neuroscience 8: 175.

Diamond, Adele. 2013. "Executive functions." Annual review of Psychology 64: 135-68.

Diehl, Rolf R. 2005. "Vasovagal syncope and Darwinian fitness." Clinical autonomic research 15(2): 126-9.

Fellows, Lesley K., and Martha J. Farah. 2007. "The role of ventromedial prefrontal cortex in decision making: judgment under uncertainty or judgment per se?" Cerebral cortex 17(11): 2669-74.

Frewen, Paul A., and Ruth A. Lanius. 2006. "Toward a psychobiology of posttraumatic self-dysregulation: reexperiencing, hyperarousal, dissociation, and emotional numbing." In The Psychobiology of Post-Traumatic Stress Disorder, Sep, 2005, Mount Sinai School of Medicine, 110-24. New York, NY: US. Blackwell Publishing.
Fuster, Joaquín M. 1999. "Synopsis of function and dysfunction of the frontal lobe." Acta Psychiatrica Scandinavica 99(Supplement 395): 51-7.

Fuster, Joaquín M. 2000. "Prefrontal neurons in networks of executive memory." Brain research bulletin 52(5): 331-6.

Graziano, Michael SA, and Dylan F. Cooke. 2006. "Parieto-frontal interactions, personal space, and defensive behavior." Neuropsychologia 44(6): 845-59.

Gross, James J. 2014. "Emotion regulation: Conceptual and empirical foundations." In Handbook of emotion regulation edited by James J. Gross, 3-20. New York, NY: The Guilford Press.

Groves, Alan, Chani Traube, and Gabrielle Silver. 2016. "Detection and management of delirium in the neonatal unit: a case series." Pediatrics 137(3).

iHediger, Heini. 1950. Wild Animals in Captivity London, UK: Butterworths Scientific Publications.

Heilman, Renata M., Liviu G. Crişan, Daniel Houser, Mircea Miclea, and Andrei C. Miu. 2010. "Emotion regulation and decision making under risk and uncertainty." Emotion 10(2): 257-65.

Holmes, Emily A., Richard J. Brown, Warren Mansell, R. Pasco Fearon, Elaine CM Hunter, Frank Frasquilho, and David A. Oakley. 2005. "Are there two qualitatively distinct forms of dissociation? A review and some clinical implications." Clinical psychology review 25(1): 1-23.

Joëls, Marian, Guillen Fernandez, and Benno Roozendaal. 2011. "Stress and emotional memory: a matter of timing." Trends in cognitive sciences 15(6): 280-8.

Kellgren, J.H., A. J. McGowan, and D. R. Wood. 1946. Effect of d-tubocurarine chloride on sensation in man. British medical journal 2(4484): 898.

Koutsikou, Stella, Richard Apps, and Bridget M. Lumb. 2017. “Top down control of spinal sensorimotor circuits essential for survival." The Journal of Physiology 595(13): 4151-58

Kozlowska, Kasia, Peter Walker, Loyola McLean, and Pascal Carrive. 2015. "Fear and the defense cascade: clinical implications and management." Harvard review of psychiatry 23(4): 263-87.

Lanius, Ulrich F., Sandra L. Paulsen, and Frank M. Corrigan. 2014. "Dissociation: Cortical Deafferentation and the Loss of Self." In Neurobiology and treatment of traumatic dissociation: Towards an embodied self edited by Ulrich F. Lanius, Sandra L. Paulsen, and Frank M. Corrigan, 5-28. New York: Springer Publishing Company.

LeDoux, Joseph E. 2000. "Emotion circuits in the brain." Annual review of neuroscience 23(1): 155-84.

LeDoux Joseph E. 2014. "Coming to Terms with Fear." Proceedings of the National Academy of Sciences 111 (8): 2871-8.

LeDoux, Joseph E. and Daniel S. Pine. 2016. "Using Neuroscience to Help Understand Fear and Anxiety: A Two-System Framework." American Journal of Psychiatry 173 (11): 1083-93.

Lloyd, Donna M. 2009. "The space between us: A neurophilosophical framework for the investigation of human interpersonal space." Neuroscience \& Biobehavioral Reviews 33(3): 297-304.

Lupien, Sonia J., and Martin Lepage. 2001. "Stress, memory, and the hippocampus: can't live with it, can't live without it." Behavioural brain research 127(1-2): 137-58

Lupien, Sonia J., Francoise Maheu, Mai Tu, Alexandra Fiocco, and Tania E. Schramek. 2007. "The effects of stress and stress hormones on human cognition: Implications for the field of brain and cognition." Brain and cognition 65(3): 20937.

Mason, John W. 1968. "A review of psychoendocrine research on the pituitary-adrenal cortical system." Psychosomatic medicine 30(5): 576-607. 
McConnell, Mark, and Daved van Stralen. 1997. "Emergency Medical Decision Making in the Tactical Environment." Tactical Edge Journal 15 (3): 32-9.

McEwen, Bruce S. "Mood disorders and allostatic load." Biological psychiatry 54, no. 3 (2003): 200-7.

McEwen, Bruce S., and John C. Wingfield. 2003. "The concept of allostasis in biology and biomedicine." Hormones and behavior 43(1): 2-15.

Miyake, Akira, Naomi P. Friedman, Michael J. Emerson, Alexander H. Witzki, Amy Howerter, and Tor D. Wager. 2000. "The unity and diversity of executive functions and their contributions to complex "frontal lobe" tasks: A latent variable analysis." Cognitive psychology 41(1): 49-100.

Mobbs, Dean, Predrag Petrovic, Jennifer L. Marchant, Demis Hassabis, Nikolaus Weiskopf, Ben Seymour, Raymond J. Dolan, and Christopher D. Frith. 2007. "When fear is near: threat imminence elicits prefrontal-periaqueductal gray shifts in humans." Science 317(5841): 1079-83

Murty, Vishnu P., Maureen Ritchey, R. Alison Adcock, and Kevin S. LaBar. 2010. "fMRI studies of successful emotional memory encoding: A quantitative meta-analysis." Neuropsychologia 48(12): 3459-69.

Nonnekes, Jorik, Mark G. Carpenter, J. Timothy Inglis, Jacques Duysens, and Vivian Weerdesteyn. 2015. What startles tell us about control of posture and gait." Neuroscience \& Biobehavioral Reviews 53: 131-8.

Novaco, Raymond W., Irwin G. Sarason, Thomas M. Cook, Gregory L. Robinson, and Francis J. Cunningham. 1979. "Psychological and Organizational Factors Related to Attrition and Performance in Marine Corps Recruit Training." Arlington, VA: Office of Naval Research.

Oatley, Keith, and Philip N. Johnson-Laird. 2014. "Cognitive approaches to emotions." Trends in cognitive sciences 18(3): 134-40.

Ochsner, Kevin N., and James J. Gross. 2008. "Cognitive emotion regulation: Insights from social cognitive and affective neuroscience." Current directions in psychological science 17(2): 153-8.

Paget, Marianne A. 1988. The unity of mistakes: A phenomenological interpretation of medical work. Philadelphia, PA: Temple University Press.

Panksepp Jaak, Fuchs Thomas, and lacobucci Paolo. 2011. "The basic neuroscience of vis experiences in mammals: the case of subcortical FEAR circuitry and implications for clinical anxiety." Applied Animal Behaviour Science 129(1): 1-17.

Quintana, Javier, and Joaquin M. Fuster. 1999. "From perception to action: temporal integrative functions of prefrontal and parietal neurons." Cerebral Cortex 9(3): 213-21.

Roelofs, Karin. 2017. "Freeze for action: neurobiological mechanisms in animal and human freezing." Philosophical Transactions of the Royal Society B: Biological Sciences 372;1718: 20160206

Sapolsky, Robert M. 2004. Why zebras don't get ulcers: The acclaimed guide to stress, stress-related diseases, and coping. New York, NY: Holt Paperbacks.

Shemmell, Jonathan. 2015. "Interactions between stretch and startle reflexes produce task-appropriate rapid postural reactions." Frontiers in integrative neuroscience 9(2):1-7

Sherman, Gary D., Jooa J. Lee, Amy JC Cuddy, Jonathan Renshon, Christopher Oveis, James J. Gross, and Jennifer S. Lerner. 2012. "Leadership is associated with lower levels of stress." Proceedings of the National Academy of Sciences 109(44): 17903-7.

Shtulman, Andrew, and Joshua Valcarcel. 2012. "Scientific knowledge suppresses but does not supplant earlier intuitions." Cognition 124(2): 209-15.

Shtulman, Andrew, and Kelsey Harrington. 2016. "Tensions between science and intuition across the lifespan." Topics in
Cognitive Science 8(1): 118-37.

Shtulman, Andrew, and Andrew Young. 2019. "Why do logically incompatible beliefs seem psychologically compatible?" In What is Scientific Knowledge?: An Introduction to Contemporary Epistemology of Science edited by Kevin McCain and Kostas Kampourakis 163-78. New York, NY: Routledge.

Smith, Scott M., Hugh O. Brown, James E. P. Toman, and Louis S. Goodman. 1947. "The lack of cerebral effects of d-Tubocurarine." Anesthesiology 8(1), 1-14.

Traube, Chani, Gabrielle Silver, Linda M. Gerber, Savneet Kaur, Elizabeth A. Mauer, Abigail Kerson, Christine Joyce, and Bruce M. Greenwald. 2017. "Delirium and mortality in critically ill children: epidemiology and outcomes of pediatric delirium." Critical care medicine 45(5): 891-8

VallsSolé, Josep, John C. Rothwell, Fatima Goulart, Giovanni Cossu, and Esteban Munoz. 1999. "Patterned ballistic movements triggered by a startle in healthy humans." The Journal of physiology 516(3): 931-8.

van Stralen, Daved. 2020. "Pragmatic High-Reliability Organization (HRO) during Pandemic COVID-19." Neonatology Today 15(4): 1-9.

van Stralen, Daved, Spencer Byrum and Bahadir Inozu. 2017. High Reliability for a Highly Unreliable World: Preparing for Code Blue through Daily Operations in Healthcare. North Charleston, SC: CreateSpace Publishing.

van Stralen, Daved, and William Gambino. 2020. "Error as a Faulty Failure Signal." Neonatology Today, 15(9):114-7.

van Stralen, Daved, Sean D. McKay, Thomas A. Mercer. 2020a. "Flight Decks and Isolettes: High-Reliability Organizing (HRO) as Pragmatic Leadership Principles during Pandemic COVID-19." Neonatology Today 15(7): 113-22.

van Stralen, Daved, Sean D. McKay, Thomas A. Mercer. 2020b. "Pragmatic Leadership Practices in Dangerous Contexts: High-Reliability Organizing (HRO) for Pandemic COVID-19." Neonatology Today, 15(8): 109-17.

van Stralen, Daved, and Thomas A. Mercer. 2015. "Ambiguity in the Operator's Sense." Journal of Contingencies and Crisis Management 23(2): 54-58.

van Stralen, Daved, and Thomas A. Mercer. 2020. "Pragmatic High Reliability Organizations (HRO) Modulate the Functions of Stress and Fear Behaviors during Pandemic COVID-19: The stress-fear-threat cascade." Neonatology Today, 15(10): 126-34.

Watson, Thomas C., Stella Koutsikou, Nadia L. Cerminara, Charlotte R. Flavell, Jonathan Crook, Bridget M. Lumb, and Richard Apps. 2013. "The olivo-cerebellar system and its relationship to survival circuits." Frontiers in neural circuits 7: 72 .

Whitehead, Alfred North. 1926 [1967]. Science and the Modern World, (Lowell Institute Lectures 1925), Cambridge, UK: Cambridge University Press. Reprinted New York, NY: The Free Press.

Yerkes, Robert M., and John D. Dodson. 1908. "The relation of strength of stimulus to rapidity of habit-formation." Journal of Comparative Neurology and Psychology 18: 459-82.

Disclosure: The authors have no disclosures.

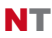




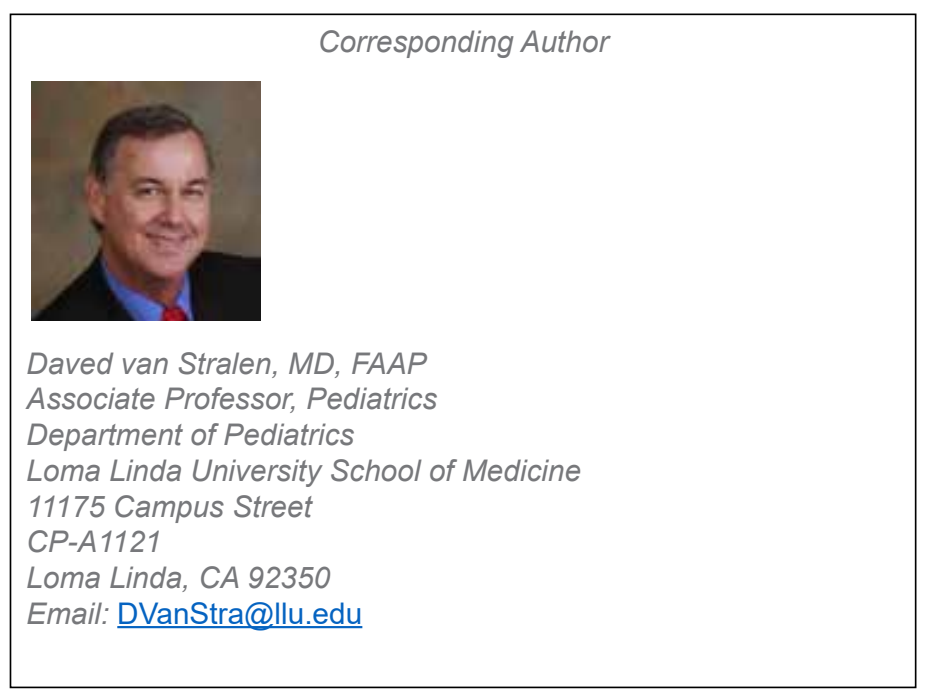

\section{Acknowledgments}

Raymond Novaco, Professor, Psychology and Social Behavior, School of Social Ecology, University of California, Irvine, California

Karl Weick- review and editing, Rensis Likert Distinguished University Professor of Organizational Behavior and Psychology, Emeritus, University of Michigan

William J. Corr, formerly with the Los Angeles City Fire Department, now deceased

Errol van Stralen, Ancora Education

Sean D. McKay, Element Rescue, LLC

William Gambino, CIV, DoD

Roger Bush, a former commissioner on the Joint Commission, and chair of its "Achieving High-Reliability Task Force."

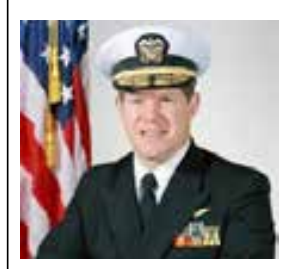

Thomas A. Mercer

Rear Admiral

United States Navy (Retired)

New subscribers are always welcome! NEONATOLOGY TQDAY

To sign up for free monthly subscription, just click on this box to go directly to our subscription page
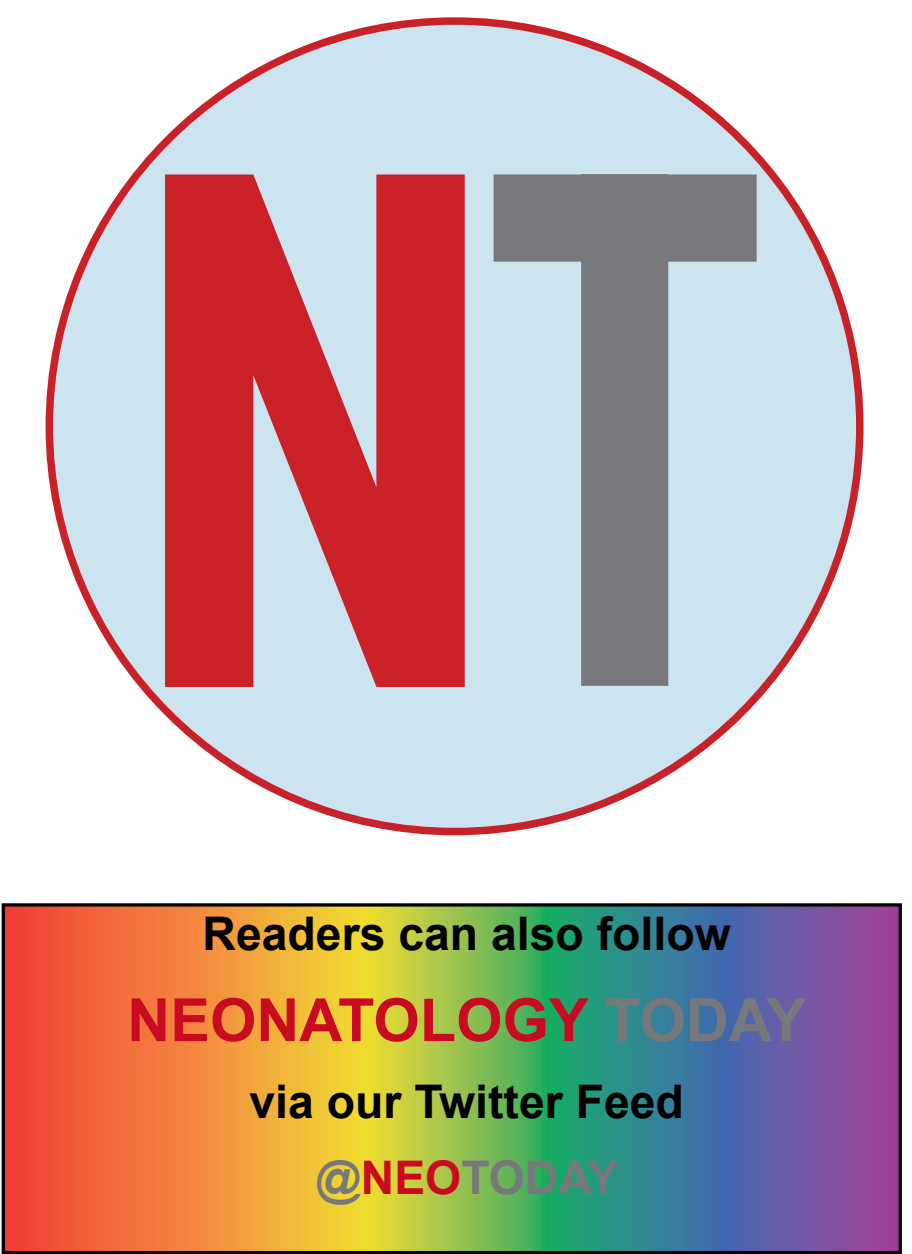

THE

BRETT TASHMAN

The Brett Tashman Foundation is a 501@(3) public charity. The mission of the Foundation is to find a cure for Desmoplastic Small Cell Round Tumors

(DSRCT). DSRCT is an aggressive pediatric cancer for which there is no cure and no standard treatment. 100 percent of your gift will be used for research.

There is no paid staff. To make your gift or for more information, go to

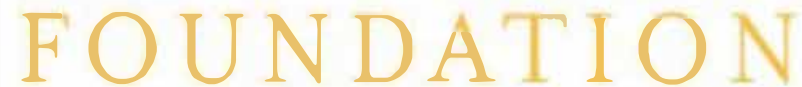

"TheBrettTashmanFoundation.org" or phone (909) 981-1530. 